\title{
Fatty acid analysis of phytopathogenic coryneform bacteria
}

\author{
Paul J. Henningson and Neil C. Gudmestad* \\ Department of Plant Pathology, North Dakota State University, Fargo, North Dakota 58105, USA
}

(Received 26 June 1990; revised 26 September 1990; accepted 1 October 1990)

\begin{abstract}
The classification of plant-pathogenic coryneform bacteria has been revised many times. All plant-pathogenic coryneform bacteria were once classified in the genus Corynebacterium. Currently, all are classified in the genera Arthrobacter, Clavibacter, Curtobacterium or Rhodococcus. Difficulties still remain regarding the characterization of species and subspecies within these groups. Analysis of cellular fatty acids by high-resolution capillary gas chromatography has recently become a standard method of bacterial identification in many laboratories. In the studies reported here, cellular fatty acid analyses were performed to examine the plant-pathogenic coryneform bacteria as an autonomous group. A total of 189 bacterial strains were profiled. Results from these studies support the division of plant-pathogenic coryneforms into four major groups (genera). Further subdivision was not required for Rhodococcus and Arthrobacter, as each genus contains only one species of plant-pathogenic bacteria. Some, but not all, Curtobacterium pathovars could be differentiated from one another based on fatty acid analysis alone. Infrasubspecific classification of plant-pathogenic Curtobacterium organisms appears to be appropriate. Most of the species or subspecies belonging to the genus Clavibacter could be differentiated using fatty acid profiles. Other chemotaxonomic methods also differentiate organisms within Clavibacter. Reclassification is warranted within Clavibacter michiganense. Clavibacter michiganense subspecies should be returned to full species status.
\end{abstract}

\section{Introduction}

The taxonomic scheme of the plant-pathogenic coryneform bacteria has undergone several revisions, many occurring within the last 10 years. These changes have been reviewed in detail by Davis (1986).

Among the revisions was the proposal of a pathovar system (Dye \& Kemp, 1977) retaining all of the species in the genus Corynebacterium; and the reclassification of certain species into Arthrobacter (Collins et al., 1981), Curtobacterium (Collins \& Jones, 1983) and Rhodococcus (Goodfellow, 1984). Davis et al. (1984) described a new genus, Clavibacter, and reclassified the remaining corynebacterial plant pathogens in this genus.

Revision of the plant-pathogenic coryneform bacteria has been based on a number of methods, e.g. morphology, physiology, biochemistry, numerical analysis, and most recently, chemotaxonomy. However, to date, no study has used fatty acid analysis to examine these organisms as an autonomous group. The use of fatty acid analysis in the identification and taxonomy of bacteria has been well-documented (Bousfield et al., 1983; Bowie et al., 1972; Collins, 1983; Minnikin et al., 1978; Moss, 1981). In this paper, we present the results of a study using fatty acid analysis to differentiate the plantpathogenic coryneform bacteria.

\section{Methods}

Identification of organisms. Organisms used in the study are listed in Table 1 . Their identities were confirmed using standard biochemical and physiological tests, including: Gram stain, gross colony colour, utilization of organic acids, production of lipase and gelatinase, growth on semi-selective media, production of acid from carbohydrates, and motility. All tests were repeated for each individual strain to assess the reliability of the test results. Gram stains were made of each culture using standard staining procedure (Difco crystal violet, $60 \mathrm{~s}$; Difco iodine, $60 \mathrm{~s} ; 95 \%(\mathrm{v} / \mathrm{v})$ ethanol, destain until clear; Difco safranin $20 \mathrm{~s})$. Cultures on NBY medium (Difco Nutrient Broth, $8.0 \mathrm{~g}$; Difco Yeast Extract, $2.0 \mathrm{~g} ; \mathrm{K}_{2} \mathrm{HPO}_{4}, 2.0 \mathrm{~g} ; \mathrm{KH}_{2} \mathrm{PO}_{4}, 0.5 \mathrm{~g}$; glucose, $5.0 \mathrm{~g}$; $\mathrm{MgSO}_{4} .7 \mathrm{H}_{2} \mathrm{O}, 1.0 \mathrm{ml}$ of a $1.0 \mathrm{M}$ solution; Oxoid agar, $10.0 \mathrm{~g} ; \mathrm{H}_{2} \mathrm{O}$, 1.01 ) were examined for gross colour differences and the presence or absence of large amounts of mucopolysaccharide (Vidaver, 1980).

Utilization of fumaric acid and sodium acetate was determined by spot inoculation of semi-solid medium in Petri dishes $\left(\mathrm{NH}_{4} \mathrm{H}_{2} \mathrm{PO}_{4}\right.$, $0.5 \mathrm{~g} ; \mathrm{K}_{2} \mathrm{HPO}_{4}, 0.5 \mathrm{~g} ; \mathrm{MgSO}_{4} .7 \mathrm{H}_{2} \mathrm{O}, 0.2 \mathrm{~g} ; \mathrm{NaCl}, 5.0 \mathrm{~g}$; Difco Yeast Extract, $0.8 \mathrm{~g}$; Oxoid agar, $12.0 \mathrm{~g}$; Brom Thymol Blue, $0.016 \mathrm{~g}$; organic acid $2.0 \mathrm{~g} ; \mathrm{H}_{2} \mathrm{O}, 1.0 \mathrm{l} ; \mathrm{pH}, 6.8$ ) (Dye \& Kemp, 1977). Plates were examined daily for $5 \mathrm{~d}$ for colour change (green to blue). Utilization was assumed if the medium surrounding the colony turned blue, which indicated a $\mathrm{pH}$ change to a value above 7.4 . Comparison was made with inoculated control plates which lacked the organic acid in question.

The method of Sierra (1957) was used to determine the presence of lipase, with Tween 80 (polyoxyethylene (20) sorbitan monooleate) as substrate. Medium (peptone, $10.0 \mathrm{~g} ; \mathrm{NaCl}, 5.0 \mathrm{~g} ; \mathrm{CaCl}_{2} .2 \mathrm{H}_{2} \mathrm{O}, 0.1 \mathrm{~g}$; Oxoid agar, $12.0 \mathrm{~g} ; \mathrm{H}_{2} \mathrm{O}, 1.0 \mathrm{l}$; adjust $\mathrm{pH}$ to 7.4 , autoclave, then add $10 \mathrm{ml}$ sterile Tween 80 to cooled medium) in Petri dishes was spot 
Table 1. Strains investigated

\begin{tabular}{|c|c|c|}
\hline Strain & Source* & Host \\
\hline \multicolumn{3}{|c|}{ Clavibacter michiganense subsp. sepedonicum (44 strains) } \\
\hline 9850 & ATCC - New York & \\
\hline 33111 & ATCC - Canada & \\
\hline R2 & S. H. DeBoer - British Columbia & \\
\hline R5 & S. H. DeBoer - British Columbia & \\
\hline R6 & S. H. DeBoer - Idaho & \\
\hline $\mathrm{Cs} 13$ & S. H. DeBoer - New Brunswick & \\
\hline Cs14 & S. H. DeBoer - Montana & \\
\hline Cs 16 & S. H. DeBoer - New Brunswick & \\
\hline Cs106 & S. H. DeBoer - West Virginia & \\
\hline Cs 118 & S. H. DeBoer & \\
\hline SDB20 & S. H. DeBoer & \\
\hline $\begin{array}{l}3 \mathrm{NM} \\
\mathrm{Cs} 3 \mathrm{R}\end{array}$ & $\begin{array}{l}\text { S. H. DeBoer - Variant } \\
\text { S. H. DeBoer - Reisolate }\end{array}$ & \\
\hline $\begin{array}{l}\text { Cs3R } \\
\text { Cs3RC }\end{array}$ & $\begin{array}{l}\text { S. H. DeBoer - Reisolate } \\
\text { S. H. DeBoer - Reisolate }\end{array}$ & Solanum tuberosum \\
\hline BRR7 & S. H. DeBoer - British Columbia & \\
\hline INM-1 & R. G. Clarke - Idaho & \\
\hline INM-B & R. G. Clarke - Idaho & \\
\hline ME-1 & F. E. Manzer - Maine & \\
\hline SS-14 & S. A. Slack - Wisconsin & \\
\hline SS-44 & S. A. Slack - Wisconsin & \\
\hline $\mathrm{CsCA}$ & A. Vidaver - California & \\
\hline A-34 & G. A. Secor - Yugoslavia & \\
\hline 2531 & NZPDDCC - Sweden & \\
\hline 2532 & NZPDDCC - Canada & \\
\hline 2534 & NZPDDCC - USA & \\
\hline 2535 & NZPDDCC - Canada & \\
\hline 2537 & NZPDDCC - USA & \\
\hline BJ 19 & NDSU Collection - Oregon & \\
\hline ULTRA & NDSU Collection - Oregon & Beta vulgaris (seed) \\
\hline DIPPE2 & NDSU Collection - Oregon & \\
\hline MONOR & NDSU Collection - Oregon & \\
\hline Olm 17 & NDSU Collection - North Dakota & \\
\hline Colol 8 & NDSU Collection - Colorado & \\
\hline SB-65 & NDSU Collection - North Dakota & \\
\hline SB-101 & NDSU Collection - North Dakota & Beta vulgaris (root) \\
\hline SB-109 & NDSU Collection - North Dakota & \\
\hline SB-136 & NDSU Collection - North Dakota & \\
\hline Pros 16 & NDSU Collection - North Dakota & \\
\hline EA1-B & NDSU Collection - North Dakota & \\
\hline SD-1 & NDSU Collection - South Dakota & \\
\hline HI-B & NDSU Collection - Minnesota & Solanum tuherosum \\
\hline OFF & NDSU Collection - Minnesota & Solanum luberosum \\
\hline As-1 & NDSU Collection - Minnesota & \\
\hline $\mathbf{P l}$ & NDSU Collection - Minnesota & \\
\hline \multicolumn{3}{|c|}{ Clavibacter michiganense subsp. michiganense (21 strains) } \\
\hline 4450 & ATCC & Unknown \\
\hline 7430 & ATCC & Cyphomandra fragranis \\
\hline 10202 & ATCC & Lycopersicon esculentum \\
\hline 14456 & ATCC & Lycopersicon esculentum \\
\hline $\mathrm{Cm} 3$ & S. H. DeBoer & \\
\hline $\mathrm{Cm} 5$ & S. H. DeBoer & \\
\hline $\mathrm{Cm} 6$ & S. H. DeBoer & Unknown \\
\hline $\mathrm{Cm} 8$ & S. H. DeBoer & \\
\hline $\mathrm{Cm} 9$ & S. H. DeBoer & \\
\hline 1104 & L. E. Claflin & \\
\hline 549 & NZPDDCC & Lycopersicon esculentum \\
\hline 1436 & NZPDDCC - New Zealand & Lycopersicon esculentum \\
\hline 1808 & NZPDDCC - New Zealand & Cyphomandra betacea \\
\hline 1811 & NZPDDCC - New Zealand & Cyphomandra betacea \\
\hline 2355 & NZPDDCC - New Zealand & \\
\hline 2541 & NZPDDCC - United Kingdom & \\
\hline 2545 & NZPDDCC - Sicily & Lycopersicon esculentum \\
\hline 2550 & NZPDDCC - Hungary & \\
\hline 2551 & NZPDDCC - Brazil & \\
\hline 5026 & NZPDDCC - USA & Capsicum annuum \\
\hline 6726 & NZPDDCC - New Zealand & Lycopersicon esculentum \\
\hline
\end{tabular}


Table 1 (continued)

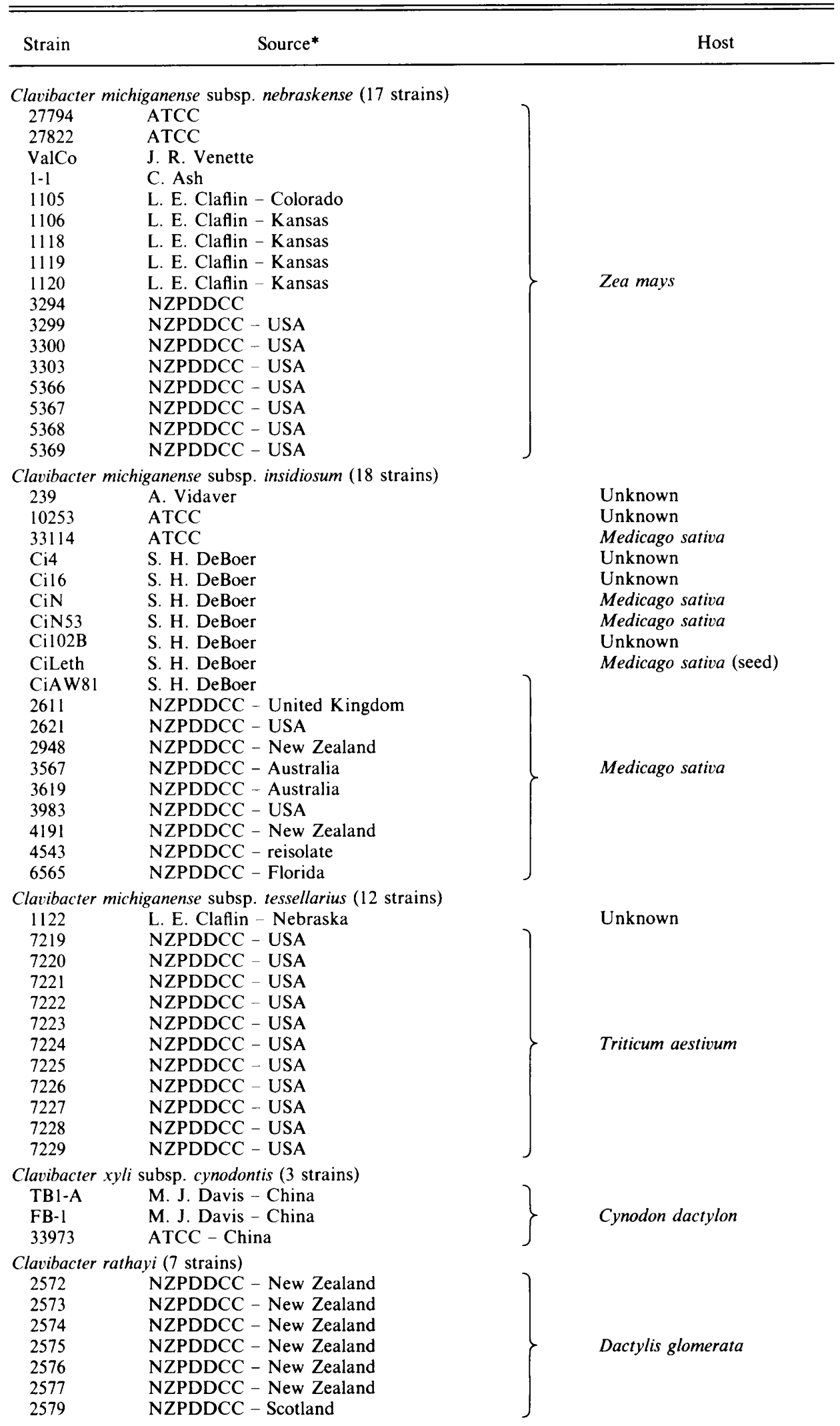


Table 1 (continued)

\begin{tabular}{|c|c|c|}
\hline Strain & Source* & Host \\
\hline \multicolumn{3}{|c|}{ Clavibacter tritici (6 strains) } \\
\hline 11402 & ATCC - India & Unknown \\
\hline 2623 & NZPDDCC - India & \\
\hline 2624 & NZPDDCC - Egypt & \\
\hline 2626 & NZPDDCC - Egypt & Triticum aestivum \\
\hline 2627 & NZPDDCC - India & \\
\hline 2628 & NZPDDCC - Iran & \\
\hline \multicolumn{3}{|c|}{ Curtobacterium flaccumfaciens pv. flaccumfaciens (17 strains) } \\
\hline 1101 & M. L. Schuster - Nebraska & Unknown \\
\hline 6887 & ATCC & Phaseolus sp. \\
\hline 2A-DK & A. Vidaver & Unknown \\
\hline CV3 & A. Vidaver & Unknown \\
\hline 2580 & NZPDDCC - USA & Phaseolus sp. \\
\hline 2581 & NZPDDCC - USA & Phaseolus sp. \\
\hline 2582 & NZPDDCC - Romania & \\
\hline 2583 & NZPDDCC & \\
\hline 2584 & NZPDDCC - Hungary & Phaseolus vulgaris \\
\hline 2585 & NZPDDCC - Dem. Rep. of Germany & \\
\hline 2588 & NZPDDCC - USA & Vigna angularis \\
\hline 2590 & NZPDDCC - USA & Vigna radiata \\
\hline 3495 & NZPDDCC - Netherlands & \\
\hline 5370 & NZPDDCC - USA & \\
\hline 5371 & NZPDDCC - USA & Phaseolus vulgaris \\
\hline 5372 & NZPDDCC - USA & \\
\hline 5373 & NZPDDCC - USA & \\
\hline \multicolumn{3}{|c|}{ Curtobacterium flaccumfaciens pv. betae (10 strains) } \\
\hline 13437 & ATCC & Unknown \\
\hline 2591 & NZPDDCC - United Kingdom & \\
\hline 2592 & NZPDDCC - United Kingdom & \\
\hline 2593 & NZPDDCC - United Kingdom & \\
\hline 2594 & NZPDDCC - United Kingdom & \\
\hline 2595 & NZPDDCC - United Kingdom & Beta vulgaris \\
\hline 4735 & NZPDDCC - United Kingdom & \\
\hline 4736 & NZPDDCC - United Kingdom & \\
\hline 4737 & NZPDDCC - United Kingdom & \\
\hline 7458 & NZPDDCC - Brazil & \\
\hline \multicolumn{3}{|c|}{ Curtobacterium flaccumfaciens pv. oortii (5 strains) } \\
\hline $6-83-70-A$ & G. A. Secor - Netherlands & Unknown \\
\hline 2632 & NZPDDCC - Netherlands & \\
\hline 3497 & NZPDDCC - Netherlands & \\
\hline 3498 & NZPDDCC - United Kingdom & Tullpa gesnerlana \\
\hline 3499 & NZPDDCC - United Kingdom & \\
\hline \multicolumn{3}{|c|}{ Curtobacterium flaccumfaciens pv. poinsettiae (10 strains) } \\
\hline 1136 & L. E. Claflin & Unknown \\
\hline 2561 & NZPDDCC - USA & \\
\hline 2563 & NZPDDCC - USA & \\
\hline 2564 & NZPDDCC - USA & \\
\hline 2565 & NZPDDCC - USA & \\
\hline 2567 & NZPDDCC - USA & Euphorbia pulcherrima \\
\hline 2568 & NZPDDCC - USA & \\
\hline 2569 & NZPDDCC - USA & \\
\hline 2570 & NZPDDCC - USA & \\
\hline 3500 & NZPDDCC - USA & \\
\hline \multicolumn{3}{|c|}{ Arthrobacter ilicis (2 strains) } \\
\hline 14264 & ATCC & Ilex opaca \\
\hline 2609 & NZPDDCC - USA & Ilex opaca \\
\hline \multicolumn{3}{|c|}{ Rhodococcus fascians (16 strains) } \\
\hline 13000 & ATCC & Lathyrus odoratus \\
\hline 2596 & NZPDDCC - United Kingdom & \\
\hline 2597 & NZPDDCC - United Kingdom & \\
\hline 2598 & NZPDDCC - United Kingdom & Fragaria $\times$ ananass $a$ \\
\hline 2599 & NZPDDCC - United Kingdom & \\
\hline 2600 & NZPDDCC - United Kingdom & Chrysanthemum $\times m c$ \\
\hline
\end{tabular}


Table 1 (continued)

\begin{tabular}{|c|c|c|}
\hline Strain & Source* & Host \\
\hline 2601 & NZPDDCC - United Kingdom & Justicia brandegeana \\
\hline 2602 & NZPDDCC - USA & Unknown \\
\hline 2603 & NZPDDCC - Canada & Unknown \\
\hline 2604 & NZPDDCC - USA & Lathyrus odoratus \\
\hline 2605 & NZPDDCC - USA & Unknown \\
\hline 5339 & NZPDDCC - United Kingdom & \\
\hline 5340 & NZPDDCC - United Kingdom & Lathyrus odoratus \\
\hline 5833 & NZPDDCC - USA & \\
\hline 7109 & NZPDDCC - Netherlands & Gladiolus sp. \\
\hline 7113 & NZPDDCC - Netherlands & Begonia sp. \\
\hline
\end{tabular}

* ATCC, American Type Culture Collection; NDSU, North Dakota State University; NZPDDCC, New Zealand Plant Disease Division Culture Collection.

inoculated and examined after $5 \mathrm{~d}$ for the presence of a cloudy precipitate surrounding the colony.

Bacteria were tested for growth on CNS and TTC media. CNS medium consisted of $1.01 \mathrm{NBY}$; nalidixic acid (freshly solubilized in $0 \cdot 1 \mathrm{M}-\mathrm{NaOH}$ ), $25.0 \mathrm{mg}$; polymyxin B sulphate ( 8000 US Pharmacopeia units $\mathrm{mg}^{-1}$ ), $32.0 \mathrm{mg}$; cycloheximide, $40.0 \mathrm{mg} ; \mathrm{LiCl}, 10 \cdot 0 \mathrm{~g} ;$ Bravo $6 \mathrm{~F}$ (tetrachloroisophthalonitrile), $0 \cdot 0625 \mathrm{ml}$; filter-sterilized ingredients were added to cooled NBY. TTC medium consisted of glucose, $10.0 \mathrm{~g}$; peptone, $10.0 \mathrm{~g}$; Casamino acids, $1.0 \mathrm{~g}$; Difco agar, $18.0 \mathrm{~g} ; \mathrm{H}_{2} \mathrm{O}, 1.0 \mathrm{l}$; $5.0 \mathrm{ml}$ of $1.0 \%(\mathrm{w} / \mathrm{v})$ solution of 2,3,5-triphenyltetrazolium chloride, autoclaved separately, was added to cooled medium (Vidaver, 1980). Suspensions of bacteria were made in test tubes containing $3.0 \mathrm{ml}$ $0 \cdot 1 \mathrm{M}$-phosphate-buffered saline. The solution was thoroughly vortexed, then $100 \mu \mathrm{l}$ volumes were placed on the medium and spread with a sterile L-shaped glass rod. Plates were examined for growth after $5 \mathrm{~d}$. Control plates which lacked the growth-inhibiting chemicals were inoculated for comparison.

Two types of media, Medium C and Difco Purple Broth Base were used to test for the production of acid from lactose, trehalose, mannitol, rhamnose and sucrose. Medium $\mathrm{C}$ consisted of $\mathrm{NH}_{4} \mathrm{H}_{2} \mathrm{PO}_{4}, 0.5 \mathrm{~g}$; $\mathrm{K}_{2} \mathrm{HPO}_{4}, 0.5 \mathrm{~g} ; \mathrm{MgSO}_{4} .7 \mathrm{H}_{2} \mathrm{O}, 0.2 \mathrm{~g} ; \mathrm{NaCl}, 5.0 \mathrm{~g}$; Difco Yeast Extract, $1.0 \mathrm{~g}$; Brom Cresol Purple, $0.7 \mathrm{ml}(1.6 \%$, w/v, alcoholic solution); Oxoid agar, $10.0 \mathrm{~g} ; \mathrm{H}_{2} \mathrm{O}, 1.01$; carbohydrate, $5.0 \mathrm{~g}$ (Dye \& Kemp, 1977). All the substrates were filter sterilized and added to cooled medium at a concentration of $0.5 \%$, then placed in sterile test tubes. Tubes were examined daily for $5 \mathrm{~d}$. Acid production was indicated by a colour change from purple to yellow.

The presence of gelatinase was tested for by stab-inoculating tubes of gelatin (Difco Nutrient Gelatin, $120.0 \mathrm{~g} ; \mathrm{H}_{2} \mathrm{O}, 1.01$ ) (Dye \& Kemp, 1977). Tubes were incubated for $10 \mathrm{~d}$ at $23 \pm 1{ }^{\circ} \mathrm{C}$.

Motility was determined by stab-inoculating tubes of soft medium (Difco Nutrient Broth, 8.0 g; Difco agar, 5.0 g) (Krieg \& Gerhardt, 1981). A heavy, cloudy transverse growth pattern from the area of inoculation after $10 \mathrm{~d}$ incubation at $23 \pm 1{ }^{\circ} \mathrm{C}$ was determined to be due to a motile culture.

Fatty acid analysis. Bacteria were stored at $-83^{\circ} \mathrm{C}$ in a freezing solution $\left(1.0 \mathrm{~g}\right.$ yeast extract, $2.28 \mathrm{~g} \mathrm{~K}_{2} \mathrm{HPO}_{4}, 0.68 \mathrm{~g} \mathrm{~K} \mathrm{H}_{2} \mathrm{PO}_{4}, 70.0 \mathrm{ml}$ $\mathrm{H}_{2} \mathrm{O}, 30.0 \mathrm{ml}$ glycerol; mix equal volumes of freezing solution and broth) until needed. Bacteria were prepared for fatty acid analysis as previously described (Gudmestad et al., 1988). Each strain was analysed once. Studies assessing the precision of fatty acid analysis were performed prior to the previous studies (Gudmestad et al., 1988). FAMEs (fatty acid methyl esters) were analysed using a gas chromatograph equipped with a flame ionization detector. A $30 \mathrm{~m} \times$ $0.25 \mathrm{~mm}$ i.d. bonded-phase fused silica capillary column (Supelco SPB-1, film thickness $0.25 \mu \mathrm{m}$ ) was used with helium as the carrier gas.
The temperature programme was initiated at $150^{\circ} \mathrm{C}$ and increased to $250{ }^{\circ} \mathrm{C}$ at $4{ }^{\circ} \mathrm{C} \mathrm{min}^{-1}$. FAME profiles were integrated and recorded using a Shimadzu C-R4A Chromatopac data processor. FAMEs were identified as previously described (Gudmestad et al., 1988; Henningson et al., 1988).

Ratios of fatty acids were calculated in all possible combinations to evaluate relative quantitative fatty acid differences. Only fatty acid ratios that allowed for the differentiation of bacterial subspecies were used for comparison and separation of the bacterial strains.

Cluster analysis of fatty acids was performed using the NTSYS-pc computer programs (Rohlf, 1988). The sIMQUAL (similarity for qualitative data) program with the Dice coefficient was chosen for data analysis. This program was derived from the statistics developed by Sneath \& Sokal (1973). Data were input in a 14 column $\times 189$ row matrix. Fatty acids were assigned the values 0 or 1 for the program based on the following scheme: $0=$ fatty acid was absent, $1=$ fatty acid was present for i14:0, a15:1, a15:0,i16:0,16:1t, X1 (unknown), $18: 2$ and $10 \mathrm{mel} 19: 0$. Fatty acids $14: 0, \mathrm{i} 15: 0$, a $17: 0$ and i1 $18: 0$ present in amounts $<1 \%=0$ and $>1 \%=1$. For $16: 0$, amounts $<10 \%=0$, $>10 \%=1$. For $18: 1 \mathrm{c}$, amounts $<5 \%=0$ and $>5 \%=1$.

\section{Results}

\section{Cultural and biochemical characteristics}

All cultures tested were Gram-positive. Cells from all species and subspecies were small and generally pleomorphic, with the exception of Rhodococcus fascians. Many of the $R$. fascians strains were long and straight, and had a small number of cells that appeared to be joined end-to-end in pairs. On NBY medium certain species exhibited very characteristic colony colours that could be used tentatively to identify the organisms. For example, Clavibacter michiganense subsp. insidiosum produced indigoidine (a deep blue and indigo mix) on the colony edges (Dye \& Kemp, 1977). Cultures of $\mathrm{Cl}$. michiganense subsp. tessellarius, $\mathrm{Cl}$. michiganense subsp. nebraskense and Curtobacterium flaccumfaciens pv. poinsettiae were orange. Clavibacter rathayi and $\mathrm{Cl}$. tritici were bright yellow while strains of Cur. flaccumfaciens 
Table 2. Biochemical and cultural characteristics of some plant-pathogenic coryneform bacteria*

\begin{tabular}{|c|c|c|c|c|c|c|c|c|c|c|c|c|c|c|c|c|c|}
\hline \multirow[b]{3}{*}{ Bacterial species } & \multirow{2}{*}{\multicolumn{2}{|c|}{$\begin{array}{l}\text { Organic } \\
\text { acids }\end{array}$}} & \multirow{2}{*}{\multicolumn{2}{|c|}{ Enzymes }} & \multirow{2}{*}{\multicolumn{2}{|c|}{$\begin{array}{l}\text { Selective } \\
\text { medium }\end{array}$}} & \multirow[b]{3}{*}{ Mot } & \multicolumn{7}{|c|}{ Acid from carbohydrate using: } & \multirow{2}{*}{\multicolumn{3}{|c|}{$\begin{array}{c}\text { Colony } \\
\text { characteristics }\end{array}$}} \\
\hline & & & & & & & & \multicolumn{3}{|c|}{$\begin{array}{l}\text { Purple Broth } \\
\text { plus }\end{array}$} & \multicolumn{4}{|c|}{$\begin{array}{l}\text { Medium } C \\
\text { plus }\end{array}$} & & & \\
\hline & $\mathrm{Fm}$ & $\mathrm{Na}$ & $\mathrm{T} 80$ & Gel & CNS & TTC & & Man & Rha & Suc & Lac & Tre & Man & Rha & Ind & Ep & Colour $\dagger^{2}$ \\
\hline A. ilicis & $v$ & + & - & + & + & + & $\mathrm{v}$ & + & + & - & $\mathbf{v}$ & + & + & + & - & - & $\mathrm{Y} ; \mathrm{W} / \mathrm{Be}$ \\
\hline$C l$ michigenense subsp. & & & & & & & & & & & & & & & & & \\
\hline insidiosum & - & - & - & - & - & - & - & + & - & - & $\mathbf{v}$ & $\mathbf{v}$ & + & - & + & + & DY \\
\hline$C l . m$. subsp. michiganense & + & + & - & - & + & + & - & - & - & $v$ & $\mathrm{v}$ & + & + & - & - & - & Y;DY \\
\hline Cl. m. subsp. nebraskense & + & $\mathrm{v}$ & - & - & + & - & - & - & - & - & $\mathrm{v}$ & + & $\mathbf{v}$ & - & - & - & $\mathrm{O}$ \\
\hline Cl. rathayi & + & - & - & $\mathbf{v}$ & - & - & - & + & - & - & - & + & + & - & - & - & $\mathrm{BrY}$ \\
\hline Cl. m. subsp. sepedonicum & $\mathrm{v}$ & $\mathrm{v}$ & - & - & - & - & - & + & - & + & - & - & + & - & - & - & $\mathbf{W} / \mathbf{B e}$ \\
\hline $\mathrm{Cl} . \mathrm{m}$. subsp. tessellarius & + & + & + & - & + & + & - & - & - & $v$ & + & + & + & - & - & - & $\mathrm{O}$ \\
\hline Cl. tritici & + & $\mathrm{v}$ & + & - & + & - & - & + & - & + & - & $\mathrm{v}$ & + & - & - & - & $\mathrm{BrY}$ \\
\hline Cur. flaccumfaciens pv. betae & - & + & + & $\mathrm{v}$ & + & + & + & - & + & $\mathbf{v}$ & + & + & + & + & - & - & Y;DY \\
\hline Cur. fl. pv. flaccumfaciens & + & $\mathrm{v}$ & + & $\mathrm{v}$ & + & + & $\mathrm{v}$ & - & $\mathbf{v}$ & $\mathrm{v}$ & $\mathrm{v}$ & + & + & + & - & $\mathrm{v}$ & $\mathbf{Y} ; \mathbf{V}$ \\
\hline Cur. fl. pv. oortii & - & + & + & + & + & + & + & - & + & $\mathrm{v}$ & + & + & + & + & - & - & $\mathbf{Y} ; \mathbf{V}$ \\
\hline Cur. $f$. pv. poinsettiae & - & + & + & $\mathrm{v}$ & + & + & v & - & - & $\mathbf{v}$ & + & + & + & - & - & - & $\mathrm{O} ; \mathrm{Y} / \mathrm{O}$ \\
\hline R. fascians & + & + & + & - & - & + & - & - & - & - & - & + & + & - & - & - & $\mathrm{Y} / \mathrm{O} ; \mathrm{Br} Y$ \\
\hline
\end{tabular}

,$+ 80-100 \%$ of organisms positive;,$- 80-100 \%$ of organisms negative; $v$, organisms were variable either between strains or when test was repeated.

* Abbreviations: Fm, fumaric acid; Na, sodium acetate; T80, lipase for Tween 80; Gel, gelatinase; CNS, growth on CNS; TTC, growth on TTC; Mot, motility; Man, mannitol; Rha, rhamnose; Suc, sucrose; Lac, lactose; Tre, trehalose; Ind, presence of indigoidine; Ep, presence of extracellular pigments.

† Colony colour on NBY: DY, dull yellow; Y, yellow; O, orange; BrY, bright yellow; Y/O, yellow-orange; W/Be, white/beige; V, variable.

pv. oortii were yellow. Most cultures of $\mathrm{Cl}$. michiganense subsp. sepedonicum were a pale white/beige colour and formed a distinct pattern on the medium. A deep blue extracellular pigment was found in two cultures of Cur. flaccumfaciens pv. flaccumfaciens. Purple-coloured strains of Cur. flaccumfaciens have been described and named Cur. flaccumfaciens var. violaceum (Shuster et al., 1968). The vast majority of species and subspecies of Clavibacter produced small to moderate amounts of mucopolysaccharide in culture, in contrast to Curtobacterium strains, which were nearly all non-mucoid.

Results from the biochemical tests are presented in Table 2. Average test scores for each test for each species were determined by the number of strains that were positive for that particular trait. Percent positive scores were transcribed to $+(80-100 \%),-(0-20 \%)$ or $\mathrm{V}$ (variable among strains or when the test was repeated with conflicting results).

No cultures produced acid from lactose or trehalose using the Difco Purple Broth Base test. All cultures were positive for acid production from sucrose on Medium C.

\section{Fatty acid composition}

A total of 24 fatty acids were detected from bacterial samples, of which 19 were in quantities large enough to be identified (Table 3). Ranges and means of fatty acid amounts for each genus are listed in Table 3. Means for each genus were calculated using mean values of each species within that genus.

Rhodococcus can be separated from the other genera by the presence of $14: 0,15: 0,16: 1 \mathrm{t}, 16: 0, \mathrm{X} 1,18: 1 \mathrm{c}$ and 10me 19:0 fatty acids (Table 3). These fatty acids are absent or greatly diminished in Clavibacter, Curtobacterium and Arthrobacter. Rhodococcus can also be separated by the absence or small amounts of i14:0, i15:0, i16:0, a17:0 and i18:0 compared to greater amounts of these fatty acids in the Clavibacter, Curtobacterium and Arthrobacter species.

Arthrobacter can be separated from Clavibacter by comparing mean amounts of $14: 0$, al $15: 1$, a $15: 0, \mathrm{i} 16: 0$, a17:0 and i18:0 (Table 3), and from Curtobacterium by comparing mean amounts of i14:0, i15:0, a15:0, a17:0 and i18:0. Clavibacter and Curtobacter species can be differentiated by comparing mean amounts of a15:1, i15:0, i16:Q a17:0 and i18:0.

Values are given for minimum, maximum, mean and standard deviation of each major fatty acid $(>1 \%$ of total) for each species and subspecies in the genus Clavibacter in Table 4. Fatty acids a15:0, i16:0 and a17:0 constitute approximately $90 \%$ of the total fatty acid content in this genus.

Ratios of various fatty acids were calculated to differentiate species and subspecies within the genus Clavibacter (Table 5). The mean for each species listed is the mean of ratios calculated for each strain of the species. Ratios calculated were a15:0/i16:0, a17:0/116:0, a15:0/a17:0, i16:0/a15:1 and a15:1/16:0. Not all strains of $\mathrm{Cl}$. rathayi and $\mathrm{Cl}$. michiganense subsp. tessellarius, $\mathrm{Cl}$. michiganense subsp. michiganense and $\mathrm{Cl}$. michiganense subsp. nebraskense contained a15:1. There- 


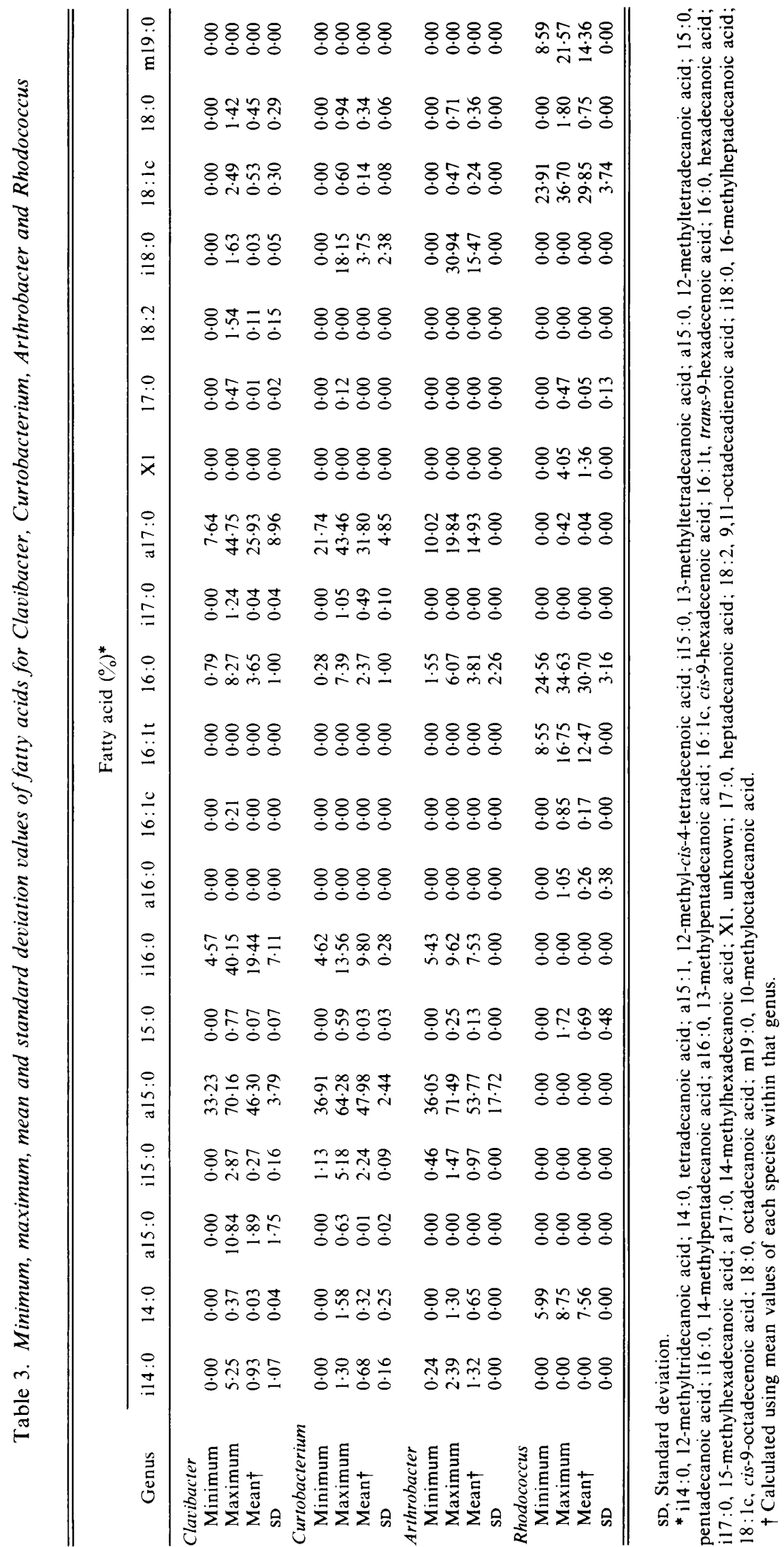


Table 4. Minimum, maximum, mean and standard deviation of major fatty acids in Clavibacter species

\begin{tabular}{|c|c|c|c|c|c|c|c|}
\hline \multirow[b]{2}{*}{ Bacterial species } & \multicolumn{7}{|c|}{ Fatty acids $(\%)^{*}$} \\
\hline & i14:0 & als:1 & a 15:0 & $\mathrm{i} 16: 0$ & $16: 0$ & a17:0 & $18: 1 \mathrm{c}$ \\
\hline \multicolumn{8}{|c|}{ Cl. xyli subsp. cynodontis } \\
\hline Minimum & 0.00 & 0.00 & $40 \cdot 79$ & $9 \cdot 17$ & $4 \cdot 17$ & 39.69 & 0.00 \\
\hline Maximum & 0.14 & 0.00 & $42 \cdot 55$ & $10 \cdot 66$ & $6 \cdot 31$ & 44.75 & $0 \cdot 32$ \\
\hline Mean & 0.05 & 0.00 & $41 \cdot 46$ & $9 \cdot 75$ & $5 \cdot 33$ & $42 \cdot 73$ & $0 \cdot 11$ \\
\hline SD & 0.07 & 0.00 & $0 \cdot 78$ & 0.65 & $0 \cdot 88$ & $2 \cdot 19$ & $0 \cdot 15$ \\
\hline \multicolumn{8}{|c|}{$\mathrm{Cl}$. michiganense subsp. michiganense } \\
\hline Minimum & 0.00 & 0.00 & $45 \cdot 78$ & $8 \cdot 36$ & 1.07 & $13 \cdot 54$ & 0.00 \\
\hline Maximum & 1.43 & $7 \cdot 35$ & $65 \cdot 53$ & $28 \cdot 89$ & $8 \cdot 27$ & $30 \cdot 57$ & 0.97 \\
\hline Mean & 0.66 & $1 \cdot 70$ & $50 \cdot 88$ & $20 \cdot 06$ & 4.00 & $20 \cdot 78$ & 0.57 \\
\hline SD & 0.32 & $1 \cdot 42$ & $4 \cdot 35$ & 4.90 & 1.87 & 3.86 & $0 \cdot 32$ \\
\hline \multicolumn{8}{|c|}{ Cl. m. subsp. sepedonicum } \\
\hline Minimum & 0.00 & 1.48 & $33 \cdot 23$ & $7 \cdot 22$ & 0.97 & 21.94 & 0.00 \\
\hline Maximum & 0.67 & $10 \cdot 84$ & $50 \cdot 32$ & $20 \cdot 70$ & $6 \cdot 18$ & $41 \cdot 04$ & $2 \cdot 49$ \\
\hline Mean & $0 \cdot 15$ & $5 \cdot 84$ & $42 \cdot 86$ & $15 \cdot 50$ & 3.02 & $29 \cdot 67$ & 0.83 \\
\hline SD & $0 \cdot 20$ & $2 \cdot 39$ & $4 \cdot 24$ & $3 \cdot 11$ & $1 \cdot 19$ & $4 \cdot 58$ & $0 \cdot 70$ \\
\hline \multicolumn{8}{|c|}{ Cl. m. subsp. nebraskense } \\
\hline Minimum & $0 \cdot 31$ & 0.00 & $42 \cdot 95$ & $12 \cdot 20$ & $1 \cdot 76$ & $14 \cdot 68$ & $0 \cdot 00$ \\
\hline Maximum & $1 \cdot 20$ & 3.98 & $52 \cdot 77$ & $28 \cdot 01$ & $6 \cdot 22$ & 34.60 & 0.74 \\
\hline Mean & 0.67 & 1.47 & $48 \cdot 14$ & $20 \cdot 38$ & $4 \cdot 22$ & $23 \cdot 75$ & $0 \cdot 24$ \\
\hline SD & $0 \cdot 22$ & 1.02 & $2 \cdot 51$ & $3 \cdot 22$ & $1 \cdot 37$ & $4 \cdot 62$ & $0 \cdot 26$ \\
\hline \multicolumn{8}{|c|}{ Cl. $m$. subsp. insidiosum } \\
\hline Minimum & 0.00 & $0 \cdot 51$ & $45 \cdot 11$ & $4 \cdot 57$ & 0.79 & $23 \cdot 81$ & $0 \cdot 00$ \\
\hline Maximum & 0.84 & $3 \cdot 76$ & $70 \cdot 16$ & $17 \cdot 82$ & $3 \cdot 59$ & $41 \cdot 85$ & $1 \cdot 38$ \\
\hline Mean & $0 \cdot 17$ & $1 \cdot 61$ & $48 \cdot 87$ & 11.46 & 1.89 & $34 \cdot 32$ & $0 \cdot 39$ \\
\hline SD & 0.22 & 0.96 & $5 \cdot 21$ & $3 \cdot 22$ & 0.71 & 4.64 & 0.45 \\
\hline \multicolumn{8}{|c|}{ Cl. m. subsp. tessellarius } \\
\hline Minimum & 0.73 & 0.00 & $45 \cdot 74$ & $20 \cdot 61$ & $2 \cdot 83$ & $16 \cdot 04$ & 0.26 \\
\hline Maximum & 1.43 & $1 \cdot 23$ & $49 \cdot 12$ & $28 \cdot 39$ & 4.47 & $25 \cdot 25$ & 0.91 \\
\hline Mean & 0.94 & $0 \cdot 74$ & $47 \cdot 49$ & $23 \cdot 86$ & $3 \cdot 76$ & 21.99 & 0.59 \\
\hline SD & $0 \cdot 19$ & $0 \cdot 36$ & 0.91 & $2 \cdot 14$ & 0.43 & $2 \cdot 26$ & $0 \cdot 16$ \\
\hline \multicolumn{8}{|l|}{ Cl. rathayi } \\
\hline Minimum & 0.80 & 0.00 & $47 \cdot 62$ & 18.05 & $2 \cdot 38$ & $20 \cdot 65$ & 0.00 \\
\hline Maximum & 1.54 & 0.95 & $51 \cdot 88$ & $26 \cdot 58$ & $3 \cdot 11$ & $24 \cdot 31$ & 0.94 \\
\hline Mean & $1 \cdot 26$ & 0.51 & $50 \cdot 15$ & $20 \cdot 62$ & $2 \cdot 74$ & $23 \cdot 15$ & 0.42 \\
\hline SD & 0.23 & 0.36 & 1.21 & 3.01 & $0 \cdot 26$ & 1.22 & 0.38 \\
\hline \multicolumn{8}{|l|}{ Cl. tritici } \\
\hline Minimum & 2.41 & 1.01 & $37 \cdot 88$ & $29 \cdot 10$ & $3 \cdot 59$ & $7 \cdot 64$ & 0.00 \\
\hline Maximum & $5 \cdot 25$ & 4.99 & $43 \cdot 36$ & $40 \cdot 15$ & 4.92 & $14 \cdot 51$ & $2 \cdot 17$ \\
\hline Mean & 3.56 & $3 \cdot 26$ & $40 \cdot 58$ & 33.90 & $4 \cdot 27$ & $11 \cdot 08$ & $1 \cdot 12$ \\
\hline SD & 0.86 & $1 \cdot 51$ & $1 \cdot 60$ & 3.53 & 0.50 & 2.06 & 0.73 \\
\hline
\end{tabular}

fore, ratios generated for these organisms using a value of zero for the amount of a15:1 as the denominator were not useful in differentiating them from other organisms.

A dichotomous key for differentiation of species and subspecies of Clavibacter was developed using ratio amounts (Table 6). The species and subspecies could be differentiated when ranges of fatty acid ratios did not overlap. Further differentiation could be accomplished when the means of fatty acid ratios were widely divergent (Table 5).

Values for the minimum, maximum, mean and standard deviation for major fatty acids for each Curtobacterium flaccumfaciens pathovar are given in Table 7. Among the Curtobacterium flaccumfaciens pathovars, pv. poinsettiae could be differentiated from pv. flaccumfaciens and pv. betae by comparing amounts of a17:0. Cur. flaccumfaciens pv. poinsettiae could be separated from pv. oortii and pv. betae by i18:0. No other direct fatty acid comparisons were useful in differentiating the Curtobacterium pathovars. Ratios developed from pairs of fatty acids were not useful in distinguishing the Cur. Alaccumfaciens pathovars.

The Rhodococcus and Arthrobacter genera each contain only one plant-pathogenic species, therefore no differentiation was necessary (Table 3 ).

In calculating similarity coefficients, fatty acid data were converted to a binary, weighted statistical method that treated fatty acids as being either present or absent 
Table 5. Fatty acid ratios in Clavibacter species

\begin{tabular}{|c|c|c|c|c|c|}
\hline \multirow[b]{2}{*}{ Bacterial species } & \multicolumn{5}{|c|}{ Fatty acid ratios* } \\
\hline & $\begin{array}{l}\text { a15:0/ } \\
\text { i16:0 }\end{array}$ & $\begin{array}{l}\text { a17:0/ } \\
\text { i16:0 }\end{array}$ & $\begin{array}{l}\text { a15:0/ } \\
\text { a17:0 }\end{array}$ & $\begin{array}{l}\text { i16:0/ } \\
\text { a15:1 }\end{array}$ & $\begin{array}{c}\text { a15:1/ } \\
16: 0\end{array}$ \\
\hline \multicolumn{6}{|c|}{ Cl. xyli subsp. cynodontis } \\
\hline Minimum & 3.99 & $3 \cdot 72$ & 0.92 & $-\dagger$ & 0.00 \\
\hline Maximum & $4 \cdot 45$ & $4 \cdot 77$ & 1.07 & - & 0.00 \\
\hline Mean & $4 \cdot 27$ & 4.42 & 0.97 & - & 0.00 \\
\hline SD & 0.20 & $0 \cdot 49$ & 0.07 & - & 0.00 \\
\hline \multicolumn{6}{|c|}{ Cl. michiganense subsp. tessellarius } \\
\hline Minimum & 1.67 & $0 \cdot 56$ & 1.90 & $19 \cdot 43$ & 0.00 \\
\hline Maximum & $2 \cdot 35$ & $1 \cdot 17$ & $2 \cdot 95$ & 34.59 & 0.31 \\
\hline Mean & 2.01 & 0.94 & $2 \cdot 19$ & $27 \cdot 55$ & 0.19 \\
\hline SD & $0 \cdot 20$ & $0 \cdot 16$ & $0 \cdot 26$ & 5.04 & $0 \cdot 10$ \\
\hline \multicolumn{6}{|c|}{ Cl. m. subsp. insidiosum } \\
\hline Minimum & 2.59 & $1 \cdot 60$ & $1 \cdot 15$ & 3.43 & 0.25 \\
\hline Maximum & $15 \cdot 35$ & $6 \cdot 23$ & 2.95 & 27.61 & 1.99 \\
\hline Mean & $4 \cdot 88$ & $3 \cdot 34$ & 1.47 & 9.89 & 0.86 \\
\hline SD & $2 \cdot 72$ & $1 \cdot 28$ & 0.38 & 6.68 & 0.43 \\
\hline \multicolumn{6}{|c|}{ Cl. m. subsp. michiganense } \\
\hline Minimum & $1 \cdot 58$ & 0.54 & 1.85 & $2 \cdot 29$ & 0.00 \\
\hline Maximum & $6 \cdot 77$ & 3.66 & 4.35 & 36.69 & 2.69 \\
\hline Mean & $2 \cdot 79$ & $1 \cdot 16$ & $2 \cdot 54$ & $14 \cdot 40$ & 0.55 \\
\hline SD & $1 \cdot 16$ & 0.63 & 0.61 & 6.88 & 0.67 \\
\hline \multicolumn{6}{|c|}{ Cl. m. subsp. nebraskense } \\
\hline Minimum & $1 \cdot 70$ & 0.69 & $1 \cdot 34$ & $5 \cdot 34$ & 0.00 \\
\hline Maximum & $4 \cdot 33$ & $2 \cdot 37$ & 3.57 & $45 \cdot 72$ & 0.72 \\
\hline Mean & 2.44 & 1.23 & $2 \cdot 12$ & $18 \cdot 16$ & 0.34 \\
\hline SD & 0.55 & 0.45 & 0.52 & 9.72 & 0.19 \\
\hline \multicolumn{6}{|c|}{ Cl. m. subsp. sepedonicum } \\
\hline Minimum & 1.90 & $1 \cdot 10$ & 0.81 & 1.29 & 0.58 \\
\hline Maximum & $6 \cdot 54$ & $5 \cdot 42$ & $2 \cdot 19$ & $11 \cdot 11$ & $8 \cdot 21$ \\
\hline Mean & 2.92 & $2 \cdot 08$ & 1.48 & $3 \cdot 24$ & $2 \cdot 37$ \\
\hline SD & 0.90 & 0.89 & 0.29 & 1.91 & 1.69 \\
\hline \multicolumn{6}{|l|}{ Cl. rathayi } \\
\hline Minimum & 1.79 & 0.78 & 2.06 & 19.08 & 0.00 \\
\hline Maximum & 2.87 & $1 \cdot 34$ & $2 \cdot 31$ & 39.88 & 0.33 \\
\hline Mean & 2.49 & $1 \cdot 15$ & $2 \cdot 17$ & 29.05 & $0 \cdot 18$ \\
\hline SD & 0.37 & $0 \cdot 20$ & 0.08 & 9.08 & 0.12 \\
\hline \multicolumn{6}{|l|}{ Cl. tritici } \\
\hline Minimum & 1.02 & $0 \cdot 19$ & $2 \cdot 79$ & $5 \cdot 83$ & 0.28 \\
\hline Maximum & $1 \cdot 39$ & 0.50 & $5 \cdot 37$ & 39.75 & $1 \cdot 12$ \\
\hline Mean & 1.21 & 0.34 & 3.81 & $15 \cdot 52$ & 0.74 \\
\hline SD & $0 \cdot 12$ & 0.09 & 0.80 & 12.07 & 0.30 \\
\hline
\end{tabular}

SD, Standard deviation.

- See Table 3 for abbreviations.

$\dagger-$, These values could not be calculated because a15:1 $=0$.

( 1 or 0 ). Some fatty acids present in minor amounts were disregarded, i.e. treated as if they were absent. Therefore, genera initially having qualitatively similar fatty acid profiles resulted in similarity coefficients from 0 to $100 \%$. For example, the genus Rhodococcus linked at $0 \%$ similarity to the three remaining genera (Fig. 1).

Curtobacterium, Clavibacter and Arthrobacter species linked at the $70 \%$ level of similarity. Four Clavibacter strains (two $\mathrm{Cl}$. xyli subsp. cynodontis, one $\mathrm{Cl}$. michiganense subsp. michiganense and one $\mathrm{Cl}$. michiganense subsp. nebraskense) clustered with the Curtobacter and Arthro- bacter genera. The remaining Clavibacter strains formed a homogeneous group and were linked together at the $84 \%$ level of similarity. Curtobacter and Arthrobacter strains linked at the $73 \%$ level, with the vast majority linked at the $88 \%$ similarity level. No definite trends were observed in subspecies relationships beyond the $88 \%$ level of similarity.

The dendogram contains 129 Clavibacter strains. Four major groups were noted above the $84 \%$ level, containing 23,61, 16 and 19 strains, respectively. Strains within groups were linked at $100 \%$. The first group 


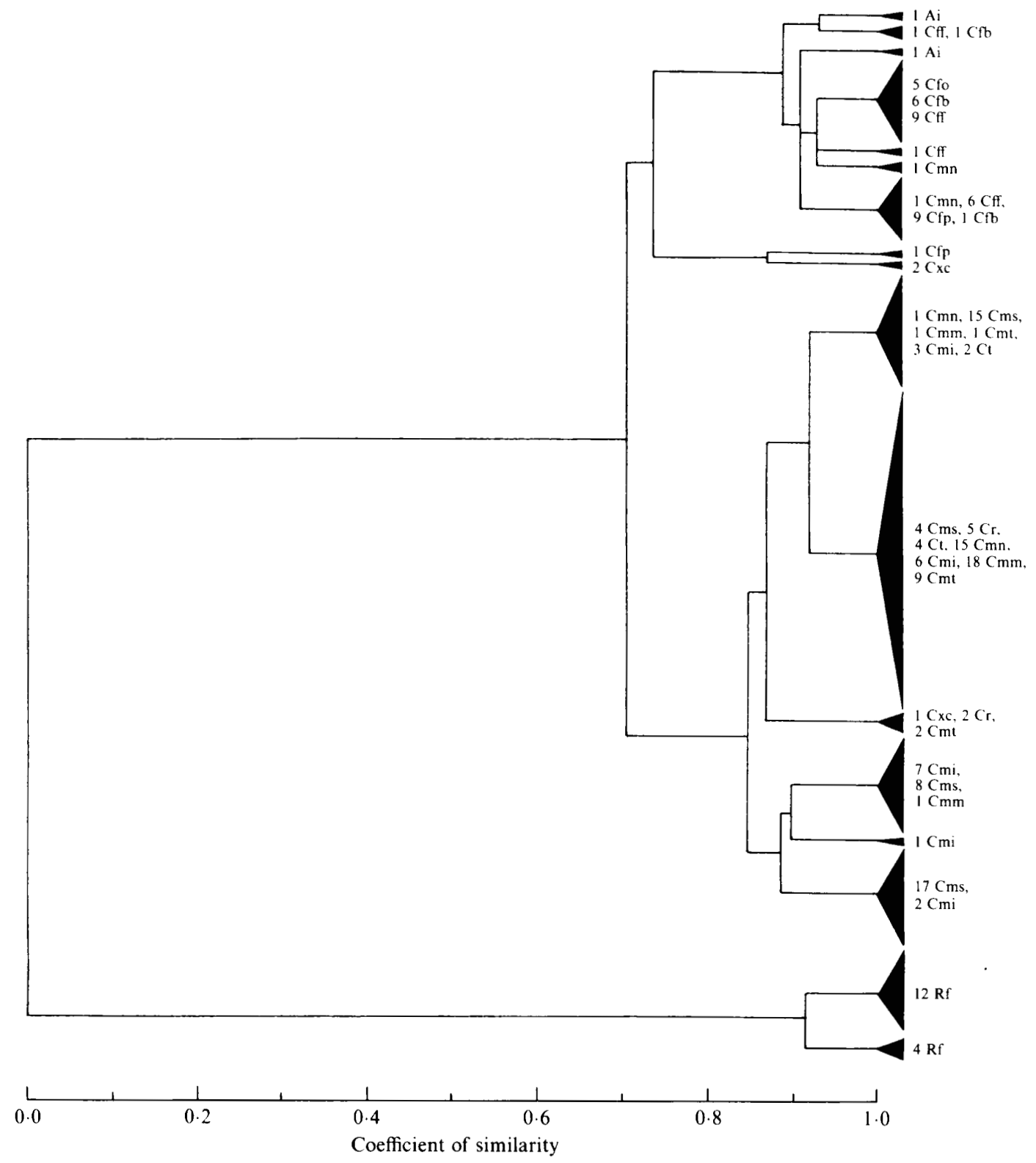

Fig. 1. Dendogram obtained by cluster analysis of similarity coefficients derived from 14 fatty acids of 189 strains of phytopathogenic coryneform bacteria. Numbers before strain abbreviations indicate the number of strains of each type located in the cluster. Ai, Arthrobacter ilicis; Rf, Rhodococcus fascians; Cfo, Cur. flaccumfaciens pv. oortii, Cff, Cur. flaccumfaciens pv. flaccumfaciens; Cfb, Cur. flaccumfaciens pv. betae; $\mathrm{Cfp}, \mathrm{Cur}$. flaccumfaciens pv. poinsettiae; $\mathrm{Ct}, \mathrm{Cl}$. tritici; $\mathrm{Cr}, \mathrm{Cl}$. rathayi; $\mathrm{Cmm}, \mathrm{Cl}$. michiganense subsp. michiganense; $\mathrm{Cmn}, \mathrm{Cl}$. michiganense subsp. nebraskense; $\mathrm{Cmi}, \mathrm{Cl}$. michiganense subsp. insidiosum; $\mathrm{Cms,} \mathrm{Cl}$. michiganense subsp. sepedonicum; $\mathrm{Cmt}, \mathrm{Cl}$. michiganense subsp. tessellarius; $\mathrm{Cxc}, \mathrm{Cl}$. xyli subsp. cynodontis.

containing 23 strains was primarily composed of $\mathrm{Cl}$. michiganense subsp. sepedonicum (15 strains) along with 8 strains of various Clavibacter subspecies. The largest group containing 61 strains was a mixture of $\mathrm{Cl}$. michiganense subsp. nebraskense (15), Cl. tritici (4), Cl. michiganense subsp. sepedonicum (4), Cl. michiganense subsp. insidiosum (6), Cl. michiganense subsp. tessellarius (9), Cl. rathayi (5) and $\mathrm{Cl}$. michiganense subsp. michiganense (18). This group contained the majority ( $85 \%$ ) of $C l$. michiganense subsp. michiganense strains and the major- ity ( $88 \%$ ) of $C l$. michiganense subsp. nebraskense strains. The group containing 16 strains was composed of approximately one half $\mathrm{Cl}$. michiganense subsp. sepedonicum and one half $\mathrm{Cl}$. michiganense subsp. insidiosum. The last major group was composed of $\mathrm{Cl}$. michiganense subsp. sepedonicum (89\%) and $\mathrm{Cl}$. michiganense subsp. insidiosum $(11 \%)$.

Two small groups containing 1 and 5 strains ( 1 strain $\mathrm{Cl}$. michiganense subsp. insidiosum, and 1 strain $\mathrm{Cl}$. xyli subsp. cynodontis, 2 strains $\mathrm{Cl}$. rathayi and 2 strains $\mathrm{Cl}$. 
Table 6. Key for the separation of Clavibacter species and subspecies using fatty acid ratios

\begin{tabular}{|c|c|}
\hline $\begin{array}{l}\text { A1: a15:0/i16:0<139* } \ldots \ldots \ldots \ldots \ldots \\
\text { A2: a15:0/i16:0 }>139 \ldots \ldots \ldots \ldots\end{array}$ & $\ldots \ldots \ldots \ldots \ldots \ldots$ Cl tritici \\
\hline $\begin{array}{l}\text { B1: a17:0/i16:0<134 } \ldots \ldots \ldots \ldots \ldots \ldots \\
\text { B2: a17:0/i16:0 }>134 \ldots \ldots \ldots \ldots \ldots\end{array}$ & 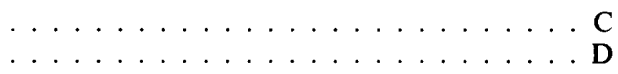 \\
\hline $\begin{array}{l}\mathrm{C} 1: \mathrm{i} 16: 0 / \mathrm{a} 15: 1<1111 \ldots \ldots \ldots \ldots \ldots \\
\mathrm{C} 2: \mathrm{i} 16: 0 / \mathrm{a} 15: 1>1111 \ldots \ldots \ldots \ldots\end{array}$ & 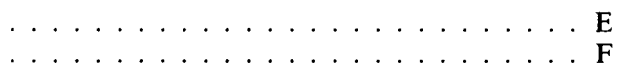 \\
\hline $\begin{array}{l}\text { D1: a15:0/a17:0<115 } \ldots \ldots \ldots \ldots \ldots \ldots \\
\text { D2: a15:0/a17:0 }>115 \ldots \ldots \ldots \ldots \ldots\end{array}$ & $\ldots \ldots \ldots \ldots \ldots \ldots \ldots \ldots$ \\
\hline 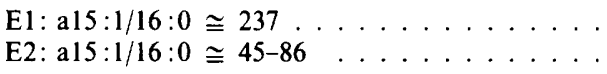 & 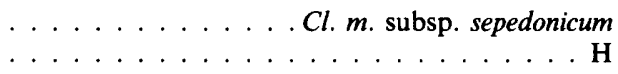 \\
\hline $\begin{array}{l}\mathrm{F} 1: \mathrm{i} 16: 0 / \mathrm{a} 15: 1 \cong 2755-2905 \quad \ldots \ldots \ldots \\
\mathrm{F} 2: \mathrm{i} 16: 0 / \mathrm{a} 15: 1 \cong 1440-1816 \quad \ldots \ldots \ldots\end{array}$ & $\begin{array}{l}\ldots l . m \text {. subsp. michiganense or subsp. nebraskense } \\
\text {. }\end{array}$ \\
\hline $\begin{array}{l}G 1: \text { a } 15: 1 / 16: 0>58 \ldots \ldots \ldots \ldots \ldots \ldots \ldots \ldots \ldots \ldots \ldots \ldots \\
G 2: \text { al } 5: 1 / 16: 0=0 \ldots \ldots \ldots\end{array}$ & 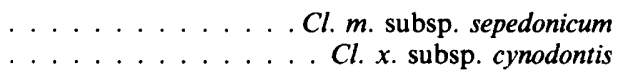 \\
\hline $\begin{array}{l}\mathrm{H} 1: \text { a } 15: 0 / \mathrm{i} 16: 0 \cong 488 \text { and } \mathrm{a} 17: 0 / \mathrm{i} 16: 0 \cong 334 \\
\mathrm{H} 2: \text { a } 15: 0 / \mathrm{i} 16: 0 \cong 261 \text { and } \mathrm{a} 17: 0 / \mathrm{i} 16: 0 \cong 120\end{array}$ & $\begin{array}{c}\ldots \ldots C l . m \text {. subsp. insidiosum } \\
\text { or } C l . m \text { m. subsp. mebraskense }\end{array}$ \\
\hline
\end{tabular}

* Values have been multiplied by 100 .

Table 7. Minimum, maximum, mean and standard deviation of major fatty acids of Curtobacterium flaccumfaciens pathovars

\begin{tabular}{|c|c|c|c|c|c|c|}
\hline \multirow{2}{*}{$\begin{array}{l}\text { Curtobacterium } \\
\text { flaccumfaciens } \\
\text { pathovar }\end{array}$} & \multicolumn{6}{|c|}{ Fatty acid $(\%)^{*}$} \\
\hline & ils:0 & a $15: 0$ & il $6: 0$ & $16: 0$ & a17:0 & i1 : :0 \\
\hline \multicolumn{7}{|l|}{ flaccumfaciens } \\
\hline Minimum & $1 \cdot 13$ & $40 \cdot 41$ & $4 \cdot 62$ & 0.28 & $22 \cdot 68$ & 0.00 \\
\hline Maximum & $5 \cdot 18$ & $63 \cdot 19$ & $13 \cdot 56$ & $5 \cdot 16$ & $33 \cdot 78$ & 14.95 \\
\hline Mean & $2 \cdot 37$ & $50 \cdot 96$ & $9 \cdot 50$ & 1.96 & $29 \cdot 49$ & 3.39 \\
\hline SD & 0.98 & $6 \cdot 37$ & $2 \cdot 57$ & $1 \cdot 30$ & $3 \cdot 22$ & 3.75 \\
\hline \multicolumn{7}{|l|}{ betae } \\
\hline Minimum & $1 \cdot 40$ & $36 \cdot 91$ & $6 \cdot 49$ & $2 \cdot 42$ & $21 \cdot 74$ & 0.84 \\
\hline Maximum & $2 \cdot 81$ & $64 \cdot 28$ & $11 \cdot 40$ & $7 \cdot 39$ & $31 \cdot 45$ & $18 \cdot 15$ \\
\hline Mean & $2 \cdot 17$ & $49 \cdot 80$ & 9.81 & $4 \cdot 04$ & $26 \cdot 16$ & 5.64 \\
\hline SD & $0 \cdot 37$ & $7 \cdot 76$ & $1 \cdot 40$ & 1.86 & $2 \cdot 85$ & $5 \cdot 52$ \\
\hline \multicolumn{7}{|l|}{ oortii } \\
\hline Minimum & 1.66 & $40 \cdot 27$ & 8.73 & $1 \cdot 33$ & 25.91 & $2 \cdot 57$ \\
\hline Maximum & $3 \cdot 25$ & $50 \cdot 65$ & $10 \cdot 79$ & $4 \cdot 36$ & $34 \cdot 97$ & 8.90 \\
\hline Mean & $2 \cdot 28$ & $45 \cdot 26$ & $10 \cdot 24$ & 2.09 & $32 \cdot 21$ & 5.97 \\
\hline SD & 0.53 & $3 \cdot 73$ & 0.78 & $1 \cdot 15$ & $3 \cdot 24$ & $2 \cdot 01$ \\
\hline \multicolumn{7}{|l|}{ poinsettiae } \\
\hline Minimum & 1.87 & $43 \cdot 44$ & 8.57 & 0.80 & 33.86 & 0.00 \\
\hline Maximum & $2 \cdot 66$ & 51.91 & $12 \cdot 02$ & $3 \cdot 52$ & 43.46 & 0.00 \\
\hline Mean & $2 \cdot 15$ & $45 \cdot 90$ & $9 \cdot 66$ & $1 \cdot 40$ & $39 \cdot 35$ & 0.00 \\
\hline SD & $0 \cdot 24$ & 2.53 & 1.03 & 0.74 & $3 \cdot 12$ & 0.00 \\
\hline
\end{tabular}

* See Table 3 for abbreviations.

michiganense subsp. tessellarius, respectively) were found.

\section{Discussion}

Reclassification of the plant-pathogenic coryneform bacteria into four groups was not proposed by a single research group, but was the cumulative result of several individual research groups (Davis et al., 1984; Collins et al., 1981; Goodfellow, 1984; Collins \& Jones, 1983). The research that resulted in the reclassification used a variety of criteria such as serology, biochemistry, physiology, cell wall sugars, cell wall proteins and peptidoglycans, menaquinones, DNA studies and lipid compositions. This is the first report using fatty acid 
profiles to compare and contrast the plant-pathogenic corynebacteria as a distinct group.

The results of the studies reported here show that the plant-pathogenic coryneform bacteria can be separated into four distinct groups based on fatty acid analysis, and support the current classification of the plant-pathogenic coryneform bacteria at the genus level. Rhodococcus fascians has large amounts of straight-chain saturated and unsaturated fatty acids and is also distinguished by the presence of tuberculostearic acid. These compounds are either not found or are present in very small quantities among species in the genera Clavibacter, Arthrobacter and Curtobacterium, which are characterized by relatively large amounts of iso- and anteisobranched chain fatty acids that can be used to differentiate the three genera. Comparison of ranges and averages of $14: 0$, a $15: 1, \mathrm{i} 15: 0$, a $15: 0, \mathrm{i} 16: 0, \mathrm{a} 17: 0$ and i18:0 can be used to delineate Arthrobacter from Clavibacter. Comparison of ranges and averages of $14: 0$, a15:1, i15:0, i16:0, i17:0, a17:0 and i18:0 can be used to delineate Clavibacter from Curtobacterium.

Cluster analysis separated the four genera into three major groups: group A, Arthrobacter and Curtobacterium; group B, Clavibacter; and group C, Rhodococcus. In group $\mathrm{A}$, no predictions were possible at any level regarding the placement of an unknown Curtobacterium or Arthrobacter strain. Any cluster within group A at the $100 \%$ level was heterogeneous, i.e. it consisted of a variety of Curtobacterium or Arthrobacter species or pathovars.

Group B contained six clusters of strains, none of which were completely homogeneous. Two major clusters were linked at the $91 \%$ level, and most others above the $85 \%$ level. No definite trends were observed, with the exception of $\mathrm{Cl}$. michiganense subsp. michiganense, in which $18(85 \%)$ strains were found in one cluster. This cluster also contained $15(88 \%)$ of the $\mathrm{Cl}$. michiganense subsp. nebraskense strains. It is apparent that on the basis of fatty acid analysis, $\mathrm{Cl}$. michiganense subsp. michiganense and subsp. nebraskense are closely related. However, these two subspecies can be separated from one another by biochemical and cultural characteristics (Table 2) and by other chemotaxonomic methods (Riley et al. 1988). Other species and subspecies of Clavibacter were distributed equally among the clusters.

Group C contained two clusters of 4 and 12 Rhodococcus fascians strains linked at the $91 \%$ level. The Rhodococcus group appeared to be completely unrelated to all other bacterial species examined. It was linked to all others at zero percent similarity. No Rhodococcus strains were found in any other cluster or group.

The fatty acid profiles of Curtobacterium flaccumfaciens pathovars were found to have a very high degree of similarity, and few comparisons of fatty acid profiles were useful in separating these pathovars from one another. Pathovars could be differentiated by biochemical tests; however, very few of these were useful. All pathovars of Cur. flaccumfaciens were essentially nonmucoid and of the same general colour. The results of this study indicate that the Cur. flaccumfaciens pathovars comprise a very homogeneous group and have few discernible differences. According to Dye et al. (1980), at the infrasubspecific level, host specificity has greater taxonomic significance than minor differences in results of biochemical or serological tests. Fatty acid analysis is inadequate for differentiating the four pathovars of $\mathrm{Cur}$. flaccumfaciens. Therefore, host-specific pathogenicity may be the only differentiating characteristic. A pathovar has been defined as a strain or set of strains with the same or similar characteristics which can be differentiated at infrasubspecific level from other strains of the same species or subspecies on the basis of distinctive pathogenicity to one or more plant hosts (Dye et al., 1980). On this basis, pathovars oortii, flaccumfaciens, betae and poinsettiae should remain in the current classification and nomenclatural system.

Davis et al. (1984) proposed the transfer of Corynebacterium iranicum, Cor. rathayi, Cor. tritici and the Cor. michiganense subspecies to a new genus, Clavibacter. Results presented here on fatty acid analysis and biochemical tests raise questions regarding the current classification of the Clavibacter michiganense subspecies. Cowan (1971) has stressed the importance of using multiple taxonomic characters and the importance of objectivity in making a classification, supported by the use of statistical or computational methods. The reclassification of Cor. michiganense, Cor. insidiosum, Cor. nebraskense, Cor. sepedonicum and Cor. tessellarius as subspecies of Cor. michiganense was proposed on the basis of a single taxonomic character, polyacrylamide gel electrophoresis (PAGE) of cell wall proteins (Carlson \& Vidaver, 1982). As little as one year prior to the reclassification, it was stated that the identity of most phytopathogenic coryneform and related bacteria could be determined only by inoculation tests in plants (Vidaver \& Starr, 1981). These authors further concluded that recent taxonomic studies have not shown any easy or certain means of identification of these bacteria below the genus level - other than their ability to cause particular plant diseases. In variance with these authors (Carlson \& Vidaver, 1982; Vidaver \& Starr, 1981), chemotaxonomic and other data existed that clearly differentiated many of the Clavibacter michiganense subspecies. Studies using serology (Lazar, 1968), cell wall amino acids (Yamada \& Komagata, 1972), DNA homology (Starr et al., 1975), numerical taxonomy (Jones, 1975) and lipid analysis (Collins \& Jones, 1980) all clearly differentiate a number of the $\mathrm{Cl}$. michiganense 
subspecies from one another.

Staley \& Krieg (1984) define bacterial species as a collection of strains that share many features in common and differ considerably from other strains. Considerable information exists on the differentiation of Clavibacter species. Clavibacter species and subspecies can be differentiated by standard biochemical and physiological tests (Vidaver, 1980; Moffett et al., 1983; this manuscript), pathogenicity tests, cultural characteristics, pigmentation and growth rate (Moffett et al., 1983). Principal cell wall amino acids can be used to differentiate $\mathrm{Cl}$. michiganense subsp. insidiosum and subsp. sepedonicum from subsp. michiganense (Yamada \& Komagata, 1972). Starr et al. (1975) differentiated $C l$. michiganense subsp. insidiosum from other $\mathrm{Cl}$. michiganense subspecies using DNA homology. $\mathrm{Cl}$. michiganense subsp. sepedonicum had a higher degree of homology to subsp. insidiosum than any other $\mathrm{Cl}$. michiganense subspecies. Lazar (1968) separated certain Clavibacter species and subspecies using serological techniques (gel diffusion of nucleoprotein antigens). Jones (1975) found many Clavibacter species and subspecies well separated from one another in her numerical taxonomic study. The fatty acid profiles of Clavibacter species and subspecies generated during the course of the studies reported here were useful in delineating these organisms from one another. In most cases, species or subspecies could be differentiated from one another by comparing ratios of fatty acids. It is apparent from these studies that fatty acid analysis of coryneform bacteria recovered from diseased plants can be a useful technique in determining the identity of a bacterium. Fatty acid analysis has been used previously in our laboratory for this purpose (Gudmestad et al., 1988).

Clavibacter michiganense subsp. sepedonicum and subsp. insidiosum each contain many copies of a unique repeated DNA sequence not found in any other Clavibacter species or subspecies (A. E. Oleson, personal communication; Mogen \& Oleson, 1987), which is in agreement with the data from Starr et al. (1975). On this basis, these subspecies are closely related to one another, but not closely related to any other $\mathrm{Cl}$. michiganense subspecies. Riley et al. (1988), using allozyme electrophoresis, found large differences between $\mathrm{Cl}$. michiganense subsp. michiganense, subsp. insidiosum and subsp. nebraskense. These differences were large enough, in their opinion, to consider each a distinct species. Based on these research findings, it would seem that the current classification of subspecies within $\mathrm{Cl}$. michiganense is far from ideal.

The classification within the genus Clavibacter appears to be incompletely resolved, and incorrect at this time. Based on the results reported here and elsewhere (Yamada \& Komagata, 1972; Collins \& Jones, 1980;
Döpfer et al., 1982; Riley et al., 1988), and in concurrence with others (Collins \& Bradbury, 1986; Riley et al., 1988), Cl. michiganense subspecies michiganense, insidiosum, sepedonicum, nebraskense and tessellarius warrant full species status in the genus Clavibacter.

This paper is published with the approval of the Director of the North Dakota Agricultural Experiment Station as Journal Article 1850.

\section{References}

Bousfield, I. J., Smith, G. L., Dando, T. R. \& HobBs, G. (1983). Numerical analysis of total fatty acid profiles in the identification of coryneform, nocardioform and some other bacteria. Journal of General Microbiology 129, 375-394.

Bowie, I. S., Grigor, M. R., Dunckley, G. G., LouitT, M. W. \& LourT, J. S. (1972). The DNA base composition and fatty acid constitution of some gram-positive pleomorphic soil bacteria. Soil Biology and Biochemistry 4, 397-412.

CaRlson, R. R. \& VIDAVER, A. K. (1982). Taxonomy of Corynebacterium plant pathogens of wheat, based on polyacrylamide gel electrophoresis of cellular proteins. International Journal of Systematic Bacteriology 32, 315-326.

Collins, M. D. (1983). Cell wall peptidoglycan and lipid composition of the phytopathogen Corynebacterium rathayi (Smith). Systematic and Applied Bacteriology 4, 193-198.

Collins, M. D. \& BradburY, J. F. (1986). Plant pathogenic species of Corynebacterium. In Bergey's Manual of Systematic Bacteriology, vol. 2, pp. 1276-1283. Edited by P. H. A. Sneath, N. S. Mair, M. E. Sharpe \& J. G. Holt. Baltimore: Williams \& Wilkins.

Collins, M. D. \& Jones, D. (1980). Lipids in the classification of coryneform bacteria containing peptidoglycans based on 2,4diaminobutyric acid. Journal of Applied Bacteriology 48, 459-470.

Collins, M. D. \& Jones, D. (1983). Reclassification of Corynebacterium flaccumfaciens, Corynebacterium betae, Corynebacterium oortii and Corynebacterium poinsettiae in the genus Curtobacterium, as Curtobacterium flaccumfaciens comb. nov. Journal of General Microbiology 129, 3545-3548.

Collins, M. D., Jones, D. \& Kroppenstedt, R. M. (1981). Reclassification of Corynebacterium ilicis (Mandel, Guba and Litsky) in the genus Arthrobacter, as Arthrobacter ilicis comb. nov. Zentralblatt für Bakteriologie, Mikrobiologie und Hygiene C 2, 318323.

Cowan, S. T. (1971). Sense and nonsense in bacterial taxonomy. Journal of General Microbiology 67, 1-8.

DAvis, M. J. (1986). Taxonomy of plant-pathogenic coryneform bacteria. Annual Review of Phytopathology 24, 115-140.

Davis, M. J., Gillaspie, A. G., Vidaver, A. K. \& Harris, R. W. (1984). Clavibacter: a new genus containing some phytopathogenic coryneform bacteria, including Clavibacter xyli subsp. xyli sp. nov., subsp. nov. and Clavibacter xyli subsp. cynodontis subsp. nov., pathogens that cause ratoon stunting disease of sugarcane and bermudagrass stunting disease. International Journal of Systematic Bacteriology 34, 107-117.

Döpfer, H., Stackebrandt, E. \& Fiedler, F. (1982). Nucleic acid hybridization studies on Microbacterium, Curtobacterium, Agromyces and related taxa. Journal of General Microbiology 128, 1697-1708.

Dye, D. W., Bradbury, M. G., Hayward, A. C., Lelliott, R. A. \& SCHROTH, M. N. (1980). International standards for naming pathovars of phytopathogenic bacteria and a list of pathovar names and pathotype strains. CMI Review of Plant Pathology 59(4), 153168.

Dye, D. W. \& KemP, W. J. (1977). A taxonomic study of plant pathogenic Corynebacterium species. New Zealand Journal of Agricultural Research 20, 563-582. 
Goodfellow, M. (1984). Reclassification of Corynebacterium fascians (Tilford) Dowson in the genus Rhodococcus fascians comb. nov. Systematic and Applied Microbiology 5, 225-229.

Gudmestad, N. C., Henningson, P. J. \& Bugbee, W. M. (1988). Cellular fatty acid comparison of strains of Corynebacterium michiganense subsp. sepedonicum from potato and sugar beet. Canadian Journal of Microbiology 34, 716-722.

Henningson, P. J., Vick, B. A., Bugbee, W. M. \& Gudmestad, N. C. (1988). Characterization of 12-methyl-cis-4-tetradecenoic acid from Corynebacterium sepedonicum. Lipids 23, 1086-1088.

JONES, D. (1975). A numerical taxonomic study of coryneform and related bacteria. Journal of General Microbiology 87, 52-96.

KRIEG, N. R. \& GerhardT, P. (1981). Solid culture. In Manual of Methods for General Bacteriology, pp. 143-150. Edited by $\mathrm{P}$. Gerhardt, R. G. E. Murray, R. N. Costilow, E. W. Nester, W. A. Wood, N. R. Krieg \& G. B. Phillips. Washington, DC: American Society for Microbiology.

LAZAR, I. (1968). Serological relationships of Corynebacterium. Journal of General Microbiology 52, 77-88.

Minnikin, D. E., Goodfellow, M. \& Collins, M. D. (1978). Lipid composition in the classification of coryneform and related taxa. In Coryneform Bacteria, pp. 85-160. Edited by I. J. Bousfield \& A. G. Callely. London: Academic Press.

MoffetT, M. L., FAHY, P. C. \& CARTWRIGHT, D. (1983). Corynebacterium. In Plant Bacterial Diseases, pp. 45-65. Edited by P. C. Fahy \& G. J. Persley. New York: Academic Press.

Mogen, B. D. \& Oleson, A. E. (1987). Homology of pCS1 plasmid sequences with chromosomal DNA in Clavibacter michiganense subsp. sepedonicum: evidence for the presence of a repeated sequence and plasmid integration. Applied and Environmental Microbiology 53, 2476-2481.

Moss, C. W. (1981). Gas-liquid chromatography as an analytical tool in microbiology. Journal of Chromatography 203, 337-347.

Riley, I. T., ReARDON, T. B. \& MCKAY, A. C. (1988). Genetic analysis of plant pathogenic bacteria in the genus Clavibacter using allozyme electrophoresis. Journal of General Microbiology 134, 3025-3030.

RoHLF, F. J. (1988). NTSYS-pc Numerical taxonomy and multivariate analysis system, version 1.40. Setauket, NY: Exeter Publishing.

Shuster, M. L., Vidaver, A. K. \& Mardel, M. (1968). A purple pigment-producing bean wilt bacterium Corynebacterium flaccumfaciens var. violaceum, $\mathrm{n}$. var. Canadian Journal of Microbiology 14, 423-427.

SiERrA, G. (1957). A simple method for the detection of lipolytic activity of micro-organisms and some observations on the influence of the contact between cells and fatty substrates. Antonie van Leeuwenhoek 23, 15-22.

SNeath, P. H. A. \& SoKal, R. R. (1973). Numerical Taxonomy. San Francisco: Freeman.

Staley, J. T. \& KRIEG, N. R. (1984). Classification of procaryotic organisms: an overview. In Bergey's Manual of Systematic Bacteriology, vol. 1, pp. 1-4. Edited by N. R. Krieg \& J. G. Holt. Baltimore: Williams \& Wilkins.

StarR, M. P., Mandel, M. \& Murata, N. (1975). The phytopathogenic coryneform bacteria in the light of DNA base composition and DNA-DNA segmental homology. Journal of General and Applied Microbiology 21, 13-26.

VIDAVER, A. K. (1980). Corynebacterium. In Laboratory Guide for the Identification of Plant Pathogenic Bacteria, pp. 12-16. Edited by N. W. Schaad. St. Paul: American Phytopathological Society.

VIDAVer, A. K. \& StaRR, M. P. (1981). Phytopathogenic coryneform and related bacteria. In The Prokaryotes, vol. 2, pp. 1880-1887. Edited by M. P. Starr, H. Stolp, H. G. Trüper, A. Balows, \& H. G. Schlegel. New York: Springer-Verlag.

Yamada, K. \& Komagata, K. (1972). Taxonomic studies on Corynebacterium bacteria: Part IV. Morphological, cultural, biochemical, and physiological characteristics. Part V. Classification of coryneform bacteria. Journal of General and Applied Microbiology 18, 399-431. 